\title{
Preliminary Experience of Autologous Free Dermal Graft Combined With Free Myocutaneous Flap in the Treatment of Refractory Bronchopleural Fistula With Empyema
}

\section{Lei Wang}

Tongde Hospital Of Zhejiang Province https://orcid.org/0000-0003-0740-5097

Zhongliang He ( $\sim$ hzpdoctor123@163.com )

Tongde Hospital of Zhejiang Province

Guoxing Chen

Tongde Hospital Of Zhejiang Province

Shunxin Xin

Tongde Hospital Of Zhejiang Province

Chun Zhang

Tongde Hospital of Zhejiang Province

\section{Research}

Keywords: bronchopleural fistula, empyema, free dermal graft, free myocutaneous flap

Posted Date: May 12th, 2021

DOI: https://doi.org/10.21203/rs.3.rs-500889/v1

License: (c) (1) This work is licensed under a Creative Commons Attribution 4.0 International License.

Read Full License 


\section{Abstract}

Background: To explore the effects of autologous free dermal graft combined with free myocutaneous flap on bronchopleural fistula with empyema.

Methods: Two patients with refractory empyema and bronchopleural fistula were treated with autologous free dermal graft combined with free myocutaneous flap. The free dermal graft was harvested from the skin around the incision and the fistula was sutured intermittently. The lateral femoral myocutaneous flap was selected as the free flap. Using microsurgery techniques, the descending branch of the lateral femoral circumflex artery and the thoracodorsal blood vessel were anastomosed intermittently to maintain the blood supply of the myocutaneous flap. After surgery, the empyema, air leakage, and the survival of the myocutaneous flap were observed.

Results: No necrosis of the myocutaneous flap was observed after surgery. There was no disease recurrence after follow-up for seven and six months, respectively. Re-examination of the chest computed tomography or magnetic resonance imaging indicated that the empyema residual cavity had disappeared.

Conclusion: Autologous free dermal graft combined with free lateral femoral myocutaneous flap transplantation is effective in the treatment of patients with bronchopleural fistula with refractory chronic empyema, with satisfactory clinical effects.

\section{Background}

Bronchopleural fistula (BPF) refers to the fistula formed between the alveoli, bronchi at various levels, and pleural cavity due to various reasons, usually occurring after lobectomy. Although BPF is relatively rare after lobectomy, the incidence is still reported to be $0.6 \%[1,2]$. With the prolongation of the disease course, patients are prone to refractory hydropneumothorax, respiratory failure, chronic empyema, and even death [3]. Owing to the existence of chronic empyema cavity that cannot be easily eliminated, treatment of BPF is difficult, especially in patients with empyema, cartilage and other necrotic tissues, where local infections are difficult to be controlled. It continues to be a problem for clinicians to completely cure the refractory BPF with empyema [4,5]. Patients often have accompanying symptoms of systemic poisoning, such as cough, anemia, hypoproteinemia, and even hemoptysis, and require longterm drainage with a tube. Although there are various approaches to treat BPF with empyema, it remains a challenge to solve the disease fundamentally. During the treatment period, the choice of surgical methods and endoscopic intervention may positively influence the treatment of individualized conditions. In any case, the goal is to control the infection, block the BPF, and effectively eliminate the empyema cavity.

Presently, in China, the treatment of BPF mostly focuses on non-surgical treatments, such as fistula closure with the endoscopic bronchial stent and injection of the sclerosing agent. However, it is very difficult to completely cure the refractory BPF with empyema by conservative treatment. The repair of BPF 
using an autologous free dermal graft may still be in the nascent stage due to the lack of literature and related data. The objective of this study was to summarize and analyze two patients with refractory BPF and empyema who were treated with autologous free dermal draft combined with free myocutaneous flap, and to evaluate the safety and effectiveness of this surgery.

\section{Methods}

A retrospective analysis was performed on two patients with BPF and refractory empyema, who received surgery at the Cardiothoracic Surgery and Orthopedics Repair and Reconstruction Center of Tongde Hospital of Zhejiang Province. Autologous free dermal draft combined with free myocutaneous flap was used in the treatment of disease. The patient characteristics are presented in Tables 1 and 2.

Table 1

Baseline characteristics

\begin{tabular}{|lllllll|}
\hline Case & $\begin{array}{l}\text { Age } \\
\text { (years) }\end{array}$ & Sex & Diagnosis & $\begin{array}{l}\text { Location of } \\
\text { bronchopleural } \\
\text { fistula }\end{array}$ & $\begin{array}{l}\text { Air leakage } \\
\text { duration } \\
\text { (years) }\end{array}$ & $\begin{array}{l}\text { Preoperative air } \\
\text { leakage } \\
\text { management }\end{array}$ \\
\hline 1 & 35 & Male & $\begin{array}{l}\text { Right } \\
\text { empyema with } \\
\text { air leakage }\end{array}$ & $\begin{array}{l}\text { Right upper } \\
\text { lobe }\end{array}$ & 2.5 & $\begin{array}{l}\text { Drainage with } \\
\text { tube }\end{array}$ \\
\hline 2 & 21 & Female & $\begin{array}{l}\text { Right } \\
\text { empyema with } \\
\text { air leakage }\end{array}$ & $\begin{array}{l}\text { Right upper } \\
\text { lobe }\end{array}$ & 1.5 & $\begin{array}{l}\text { Drainage with } \\
\text { tube }\end{array}$ \\
\hline
\end{tabular}

Table 2

Clinical characteristics

\begin{tabular}{|lllllll|}
\hline Case & $\begin{array}{l}\text { Type of bacteria } \\
\text { cultured }\end{array}$ & $\begin{array}{l}\text { Initial size of } \\
\text { empyema } \\
\text { cavity }(\mathbf{m L})\end{array}$ & $\begin{array}{l}\text { Intraoperative } \\
\text { bleeding } \\
(\mathbf{m L})\end{array}$ & $\begin{array}{l}\text { Operation } \\
\text { time (h) }\end{array}$ & $\begin{array}{l}\text { Length } \\
\text { of stay } \\
\text { (months) }\end{array}$ & $\begin{array}{l}\text { Follow- } \\
\text { up } \\
\text { (months) }\end{array}$ \\
\hline 1 & Mycobacterium & 60 & 100 & 5.5 & 6.5 & 7 \\
\hline 2 & $\begin{array}{l}\text { Stenotrophomonas } \\
\text { maltophilia }\end{array}$ & 180 & 300 & 6.2 & 1.8 & 6 \\
\hline
\end{tabular}

\section{Treatment details}

\section{Case-1}

Case- 1 was a young woman of age 35 years. One and a half years ago, she was admitted to a local hospital for multiple bullae in both lungs and pneumothorax, and underwent bullectomy in the right upper lung at the same time. Two weeks after the operation, she began to develop cough, sputum, and fever, and her condition gradually worsened. Over time, purulent exudates appeared in her chest tube, which continued to leak air. However, to aggravate matters further, there was also a pneumothorax in the left lung, and both lungs continued leaking. The patient had a history of old pulmonary tuberculosis, which had subsequently been cured, and exhibited poor lung function without a history of hypertension, 
diabetes, or other diseases. Although the patient's condition was generally stable by adjusting antibiotics and maintaining smooth drainage, what made her uncomfortable was the drainage bottle that was always accompanied by turbidity and several bubbles, and the drainage tube fixed in the chest cavity could not be pulled out in time, giving rise to irritating cough and low fever at irregular intervals. Computed tomography (CT) of the chest indicated hydropneumothorax in the right pleural cavity, simple pneumothorax in the left pleural cavity, and localized drainage tubes in both thoracic cavities (Fig. 1A). Based on her clinical symptoms and the findings of imaging, she was diagnosed to have BPF with empyema after right upper bulla resection and left spontaneous pneumothorax (compression about $30 \%$ ). Therefore, the development of a detailed treatment plan was essential. The first step was to replace the thin drainage tube on the left side of the patient with a 24F drainage tube to promote lung recruitment and then pull it out after there was no obvious air leak. In the second step, after ensuring that the left lung could function normally, we carefully examined the problem of the bronchopleural fistula with empyema on the right side. After the patient was anesthetized, bronchoscopy was performed, which confirmed that the presence of a fistula with a diameter of about $3 \mathrm{~mm}$ between the upper-right bronchus stump and the pleural cavity, and a covered bronchial stent (Boston Scientific Corporation, Natick, Massachusetts) was placed under the endoscope. After the condition was clarified, rib resection and thoracoplasty were performed simultaneously. After a part of the second rib was removed, the debridement, flushing, and removal of purulent pleural effusion and necrotic tissue were performed in the thoracic cavity. Two obvious alveolar pleural fistulae were seen on the surface of the right upper lung in the tympanic lung, with a diameter of about 2-3 mm. Using pus culture, the pathogen was identified to be Mycobacterium spp. After timely adjustment of antibiotics and adequate drainage after surgery, the condition of the patient improved. No obvious pathogenic bacteria were detected again after pus culture. Subsequently, she received a second surgery. The third step was to incise the pedicled latissimus dorsi muscle flap of a size of about $14.0 \mathrm{~cm} \times 8.0 \mathrm{~cm}$ and the serratus anterior myocutaneous flap of a size of about $10.0 \mathrm{~cm} \times$ $6.0 \mathrm{~cm}$. After the fistula was sealed using medical glue, the myocutaneous flap was sutured and fixed, and the abscess was completely packed after transposition.

Although the surgery went smoothly, the patient struggled with postoperative recovery. We discussed and agreed that the root cause was air leakage and infection. After the operation, the pedicled muscle flap also appeared to show partial atrophy, and the placed bronchial stent was coughed out of the body. The patient stayed for a long period in the hospital, during which she had undergone several debridement operations and bronchial stent closures with unsatisfactory effect. After multidisciplinary consultation, the free dermal draft combined with free myocutaneous flap was ultimately selected for the surgery. After anesthesia, the patient was placed in a semi-recumbent position. After sufficient debridement, healthy skin around the incision was selected, and the subcutaneous and epidermal tissues were removed to make a dermal graft (Fig. 1B). The graft was inserted into the two fistulas, and the exposed part was sutured and fixed with surrounding tissues. There was no obvious air leakage after the lung bulged (Fig. 1C). Later, the line connecting the right anterior superior iliac spine with the outer side of the patella was selected. Starting from the inside of the flap, the skin, subcutaneous tissue, and deep fascia were incised layer-by-layer to expose the muscle tissue. The rectus femoris and lateral femoral muscles were bluntly 
separated, and the descending branches of the lateral femoral muscle arteries and accompanying veins and nerves were separated. Ligation of the branches was distributed at the distal end of the muscle to completely free the muscle, its vascular pedicle, and nerve. The vascular pedicle was about $3 \mathrm{~cm}$ long. Finally, the pedicle was cut according to the length required by the recipient area, and a myocutaneous flap of a size of about $20 \mathrm{~cm} \times 12 \mathrm{~cm}$, composed of skin, subcutaneous tissue, fascia, and lateral femoral muscle was obtained, with the descending branch of the lateral femoral artery as the pedicle. The size of the island was about $5 \mathrm{~cm} \times 3 \mathrm{~cm}$. After the myocutaneous flap was transplanted to the recipient area and the residual cavity was filled, the descending branch of the lateral femoral artery, vein, and nerve, and the thoracic dorsal artery, vein, and nerve were intermittently sutured under the microscope using a 9-0 prolene thread to ensure the blood supply of the tissue flap and muscle activity (Fig. 1D, E). Suture between dermal graft and myocutaneous flap was also performed to enhance the vascularization to dermal graft. After the operation, the flap was sutured to normal skin around the chest without tension (Fig. 1F), and the drainage tube and drainage skin were placed. The postoperative treatment consisted of postoperative anti-infection, anti-spasm, anti-coagulation, and other treatments. The color and temperature of the myocutaneous flap were closely monitored, and the transplanted myocutaneous flap was applied to keep it warm; thus, avoiding the compression of the anastomotic wound to ensure smooth blood flow [6]. The postoperative process went smoothly, and she was discharged from the hospital after complete recovery. The chest magnetic resonance imaging (MRI) indicated that the empyema cavity and BPF were successfully sutured after surgery (Fig. 1G). During the 6-month follow-up, there was no obvious recurrence of pleural fistula and empyema.

\section{Case-2}

Case-2 was a young man of age 21 years with a history of multiple bullae in the right lung with pneumothorax. Two and a half years ago, he was admitted to a local hospital and received surgical treatment. On the third day after the operation, purulent exudates appeared in the closed drainage tube of the chest cavity, accompanied by an unpleasant smell. His main symptoms were intermittent cough and low fever. Although his fever was relieved by antibiotics and active drainage, his cough continued, and several air bubbles overflowed from the drainage bottle. The chest CT showed an abscess in the upper right chest, a gas-liquid plane in the chest cavity, a reduction in the thoracic volume, and a thickening of the pleura (Fig. 2A). Based on these observations, the patient was diagnosed to have the right BPF with empyema. After active pre-operative preparations, using the position of the original drainage tube as the appropriate incision plane, $2 \mathrm{~mm}$ BPF could be seen on the pleural surface near the top of the abscess cavity after removing the $3^{\text {rd }}, 4^{\text {th }}$, and $5^{\text {th }}$ ribs along with part of the thickened pleura, and the volume of the abscess cavity was about $180 \mathrm{~mL}$. Intraoperative debridement, flushing, and the removal of pus and necrotic tissue in the thoracic cavity were also performed. This patient had a lot of blood loss during the operation and was given a blood transfusion after the operation. During the next few weeks, anti-infection and nutritional support were the main treatment options to maintain a smooth flow. When the patient coughed actively, air leakage in the drainage bottle could be seen. The identified bacterium in the postoperative pus culture was Maltophilia stenotrophomonas, and antibiotics were adjusted in time for 
symptomatic treatment. When the patient's general condition was stable, we combined the first patient's successful experience in repairing the fistula with a dermal skin flap to evaluate whether the same approach was similarly effective. After careful pre-operative discussion and summary, considering the patient's larger abscess cavity, it was decided to directly treat the empyema with free dermal graft combined with free myocutaneous flap. The procedure was the same as that followed for the first patient, and the postoperative recovery was unremarkable. After 1.8 months of hospitalization, he was discharged from the hospital, and the incision recovered well (Fig. 2B). Re-examination of chest MRI empyema showed no recurrence and disappearance of BPF (Fig. 2C). After six months of follow-up, he lived as a healthy person.

\section{Results}

Both patients underwent staged surgery according to the above-described procedure. Overall, the treatment was satisfactory. The first patient was hospitalized for a relatively long time, which was attributed to the poor control of air leakage, in addition to the isolation and culture of mycobacteria (nontuberculous type) from the pus during the treatment process. The main component of the cell wall of these bacteria was mycolic acid, which may induce chronic infection in short-term and long-term prolonged and destructive tissue disease. Great attention has been paid to anti-infection and the choice of drainage methods. Based on the experience of case- 1 , the treatment cycle of case- 2 was significantly shortened. Although Stenotrophomonas was isolated from the pus of this patient, their overall pathogenicity was not strong. Through active anti-infection and adequate drainage, a satisfactory effect of the treatment was achieved.

For the treatment of refractory BPF with empyema, we must focus on the control of infection and completion of the surgery under the condition of smooth drainage. We applied autologous free dermal graft repair combined with free myocutaneous flap tamponade and achieved the expected results. The myocutaneous flap was observed to be free of necrosis after the operation. After several months of follow-up, there was no recurrence of the original disease. During the re-examination, chest CT or MRI indicated that the original empyema cavity disappeared, the free myocutaneous flap was active, and the clinical effect was satisfactory.

\section{Discussion}

BPF is a serious complication after lung resection and leads to adverse effects, including intractable empyema and residual lung pneumonia with the progress of the disease. BPF itself adversely affects the quality of life and survival time of patients, which is also considered to be a challenge for thoracic surgeons. Presently, the etiology and mechanism of BPF with empyema remains poorly understood. Comprehensive analysis of multiple factors has indicated that the occurrence of BPF can be summarized as a major risk factor as well as a secondary risk factor $[7,8]$. These risk factors may include: a) the manual suture technique implemented by the clinician on the bronchial stump; b) excessive removal of the lymph nodes around the bronchial stump, causing the blood supply around the stump to be blocked; 
c) excessively long bronchial stump remaining after lung resection; d) the continued presence of tumor cells in the bronchial stump; e) long-term mechanical ventilation after major surgery; f) advanced age and poor nutritional status; g) exposure to high-dose chemotherapy or radiotherapy before or after surgery [911]. Recent studies have demonstrated that pneumonectomy bears a significantly higher risk of BPF than lobectomy. This may be attributed to the fact that there is an increased risk of avascular necrosis of the bronchial stump or accumulation of secretions, leading to bacterial overgrowth and colonization and poor nutritional status $[12,13]$. Clinical studies have reported that appropriate treatment is highly important for patients showing BPF with empyema. The timeliness of the treatment is mainly reflected in avoiding persistent infection of the pleural cavity as much as possible. The common assessment methods are chest CT and bronchoscopy. The patients transitioning from the acute phase to the chronic phase are often accompanied by chronic wasting with obvious symptoms of systemic poisoning and symptoms such as cough, anemia, hypoproteinemia, and hemoptysis.

Several BPF patients with empyema were admitted in our department, who were treated with a pedicled muscle flap or free myocutaneous flap. Regarding the choice of surgical methods, we have observed that abscesses of volume less than $100 \mathrm{~mL}$ could be treated with pedicled muscle flaps, those of volume between $100 \mathrm{~mL}$ and $200 \mathrm{~mL}$ could be treated with pedicled muscle flaps, free muscle skin, or a combination of the two treatments according to intra-operative conditions, and abscess of volume more than $200 \mathrm{~mL}$ could be treated with free myocutaneous flap. Generally speaking, the treatment options for patients with refractory BPF with empyema include conservative treatment, endoscopic treatment, and surgical treatment, of which the conservative treatment mainly involves adequate chest drainage and supportive treatment (Fig. 3). However, endoscopic treatment often involves bronchoscopy occlusion, which may be more practical for most patients. However, it is difficult to treat patients with BPF, such as bronchial dislocation, severe infection in the pleural cavity, and larger fistulae, with poor efficacy of the treatment [14]. What should we do if we encounter this type of refractory BPF with empyema and a large abscess cavity? The data indicated that after full closed thoracic drainage of empyema, closure of the fistula is a key factor for the success of the subsequent operation [12]. On the other hand, our treatment plans should also take the locations of fistula and abscess cavity into consideration. If the visual field exposure of fistula is poor during the surgery, it may be difficult to be repaired by the free dermal graft. The two patients analyzed in this study both had upper lobe BPF. Based on the experiences in our center, patients with lower lobe defect had a relatively small abscess cavity, which could be treated with pedicled muscle flap alone. We have not found patients with large abscess cavity after lower lobectomy at present. Patients with lower lobe defect and large abscess cavity might be rare. Thus, although we believe the reliability of our treatment plan, evidence in patients with lower lobe defect and large abscess cavity is needed.

Generally, free dermal drafts are mainly used to repair skin and mucous membrane defects caused by burns and wounds but are relatively rarely utilized in the repair of BPF, as evident from the availability of only a few clinical reports. Autologous free dermal grafts (de-epidermized) have their unique advantages, including good flexibility and contractility, strong anti-infective ability, and abundance of surrounding capillaries. After the repair of the fistula, the air leakage was significantly reduced, and the curative effect 
exceeded expectations. After completing the repair of the fistula, we again selected free myocutaneous flaps to fill the abscess cavity. With a recent increase in the number of cases receiving tissue flaps to treat empyema, its therapeutic effect has also been unanimously recognized by thoracic surgeons. It retains the shape of the thorax and does not significantly affect the respiratory and circulatory function. Furthermore, the free myocutaneous flap does not require skin grafting due to chest wall skin defects. After the operation, the color of the flap can be observed in time to evaluate the blood supply.

Currently, the chronic empyema has been frequently treated with pedicled muscle flaps, while the free myocutaneous flaps have rarely been used, which may be due to the operation involving microscopic vascular anastomosis, surgical difficulty, and greater trauma. Pedicled muscle flaps are not applicable if the patient suffers from refractory BPF with empyema and if part of the muscle group has been severed due to previous thoracotomy or when the abscess cavity is large [15]. The free myocutaneous flap can reach almost any position in the chest cavity and can fill the contaminated cavity. In some cases, free myocutaneous flaps can also be combined with pedicled muscle flaps, pedicled pericardium slices, or pedicled omentum to treat complex empyema $[16,17]$. During the operation, we applied microsurgery techniques to use the descending branch of the lateral femoral circumflex artery of the free myocutaneous flap transplanted into the abscess cavity as the donor vessel to align with the recipient's blood vessel, and the recipient's blood can generally be from the thoracic and dorsal arteries and veins. Thoracic dorsal arteries and veins are destroyed, and intercostal vessels, intramammary vessels, and transverse cervical vessels can be selected [4]. During the operation, it is necessary to prevent the tension of the vascular pedicle and the edge of the myocutaneous flap after anastomosis, which may affect the blood supply and activity of the free myocutaneous flap.

\section{Conclusions}

In summary, the autologous free dermal graft combined with free myocutaneous flaps was an effective surgical treatment for some patients with refractory BPF with empyema. Perhaps, the number of cases in which we have applied this method to treat complicated empyema is low, and even the position of some patients with pleural fistula is not suitable for repair or is irreparable, but we had only selected the suitable cases, and the patients had recovered well in the short and mid-term. Thus, it is necessary to further clinically test this procedure on a larger patient population.

\section{Abbreviations}

BPF: bronchopleural fistula

CT: computed tomography

MRI: magnetic resonance imaging

\section{Declarations}




\section{Ethics approval and consent to participate}

All procedures carried out in this study were in accordance with the ethical standards of the institutional and national responsible committee on human experimentation and the Helsinki Declaration of 1964 and its later amendments or equivalents. This study was approved by the Ethics Committee of Tongde Hospital of Zhejiang Province. Informed consent was obtained from all individual patients included in the study.

\section{Consent for publication}

Written Informed consent for publication was obtained from all individual patients included in the study.

\section{Availability of data and materials}

The datasets used and/or analysed during the current study are available from the corresponding author on reasonable request.

\section{Competing interests}

The authors declare that they have no competing interests.

\section{Funding}

None

\section{Authors' contributions}

LW, ZH and CZ designed the study. LW collected the data. Material preparation and the data analysis were performed by LW, GC and SX. The first draft of the manuscript was written by LW. All authors read and approved the final manuscript.

\section{Acknowledgements}

None

\section{References}

1. Kashima M, Yamakado K, Takaki H, Kodama H, Yamada T, Uraki J, Nakatsuka A. Complications after 1000 lung radiofrequency ablation sessions in 420 patients: a single center's experiences. AJR Am J Roentgenol. 2011;197(4):W576-80.

2. de Baere T, Tselikas L, Woodrum D, Abtin F, Littrup P, Deschamps F, Suh R, Aoun HD, Callstrom M. Evaluating Cryoablation of Metastatic Lung Tumors in Patients-Safety and Efficacy: The ECLIPSE Trial-Interim Analysis at 1 Year. J Thorac Oncol. 2015;10(10):1468-74. 
3. Slade M. Management of pneumothorax and prolonged air leak. Semin Respir Crit Care Med. 2014;35(6):706-14.

4. Walsh MD, Bruno AD, Onaitis MW, Erdmann D, Wolfe WG, Toloza EM, Levin LS. The role of intrathoracic free flaps for chronic empyema. Ann Thorac Surg. 2011;91(3):865-8.

5. Deshpande M, Kamath A, Allinson K, Peat D, Parry W. A 76-year-old lady with chronic cough and a discharging chest wall sinus. Thorax. 2011;66(8):733, 738-739.

6. He ZH, Zhang C, Shen L, et al. Transplantation of free lateral thigh myocutaneous flap for the treatment of postoperative intractable chronic empyema. Chinese Journal of Thoracic Cardiovascular Surgery. 2016;32(7):421-2.

7. Deschamps C, Bernard A, Nichols FC 3rd, Allen MS, Miller DL, Trastek VF, Jenkins GD, Pairolero PC. Empyema and bronchopleural fistula after pneumonectomy: factors affecting incidence. Ann Thorac Surg. 2001;72(1):243-7. discussion 248.

8. Di Maio M, Perrone F, Deschamps C, Rocco G. A meta-analysis of the impact of bronchial stump coverage on the risk of bronchopleural fistula after pneumonectomy. Eur J Cardiothorac Surg. 2015;48(2):196-200.

9. Algar FJ, Alvarez A, Aranda JL, Salvatierra A, Baamonde C, López-Pujol FJ. Prediction of early bronchopleural fistula after pneumonectomy: a multivariate analysis. Ann Thorac Surg. 2001;72(5):1662-7.

10. Laperuta P, Napolitano F, Vatrella A, Di Crescenzo RM, Cortese A, Di Crescenzo V. Postpneumonectomy broncho-pleural fistula successfully closed by open-window thoracostomy associated with V.A.C. therapy. Int J Surg. 2014;12(Suppl 2):17-s19.

11. Cardillo G, Carbone L, Carleo F, Galluccio G, Di Martino M, Giunti R, Lucantoni G, Battistoni P, Batzella $\mathrm{S}$, lacono RD. The rationale for treatment of postresectional bronchopleural fistula: analysis of 52 patients. The Annals of Thoracic Surgery. 2015;100(1):251-7.

12. Fuso L, Varone F, Nachira D, Leli I, Salimbene I, Congedo MT, Margaritora S, Granone P. Incidence and Management of Post-Lobectomy and Pneumonectomy Bronchopleural Fistula. Lung. 2016;194(2):299-305.

13. De Palma A, Maruccia M, Di Gennaro F. Right thoracotomy approach for treatment of left bronchopleural fistula after pneumonectomy for tubercolosis. Gen Thorac Cardiovasc Surg. 2020;68(12):1539-42.

14. Han X, Yin M, Li L, Zhu M, Ren K, Qi Y, Li X, Wu G. Customized airway stenting for bronchopleural fistula after pulmonary resection by interventional technique: single-center study of 148 consecutive patients. Surg Endosc. 2018;32(10):4116-24.

15. Lu C, Feng Z, Ge D, Yuan Y, Zhang Y, Qi F, Gu J, Xu F. Pedicle muscle flap transposition for chronic empyema with persistent bronchopleural fistula: experience of a single clinical center in China. Surg Today. 2016;46(10):1132-7.

16. He X, He Z, Shen L, Chen G, He X. Free musculocutaneous flap transfer for refractory chronic empyema with chest wall sinus in a 43-year-old male with hemophilia A. J Thorac Dis. 
2018;10(6):E416-9.

17. Hong $X, \mathrm{He} Z$, Shen $L$, He X. Free vastus lateralis musculocutaneous flap transfer for radiationinduced chest wall fistula combined with osteomyelitis: Two case report. Medicine. 2019;98(22):e15859.

\section{Figures}
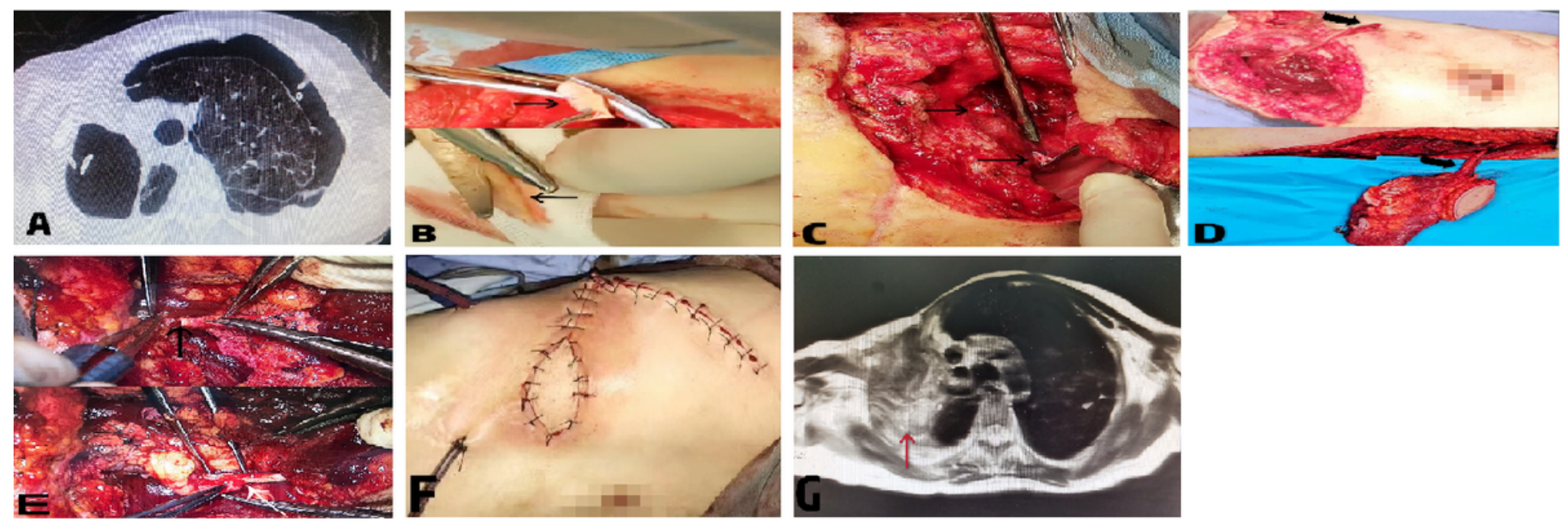

\section{Figure 1}

Clinical images of patient-1 A. The chest computed tomography at admission showed left pneumothorax and right hydropneumothorax with drainage tubes placed in the thoracic cavity on both sides. $B$. The incised skin tissue was made into a suitable dermal graft. C. The two fistulae located at the stump of the right upper lung bronchus were completely repaired with free dermal graft, without obvious air leakage (black arrow). D. Free thoracodorsal arteries, veins and nerves, and lateral femoral myocutaneous flap and its vascular pedicles and nerves (black arrow). E. Anastomosis of blood vessels and nerves during the surgery (black and white arrows). F. After surgery, the myocutaneous flap was sutured to normal skin around the chest without tension. G. The chest magnetic resonance imaging indicated that the empyema cavity and bronchopleural fistula were successfully blocked (red arrow).
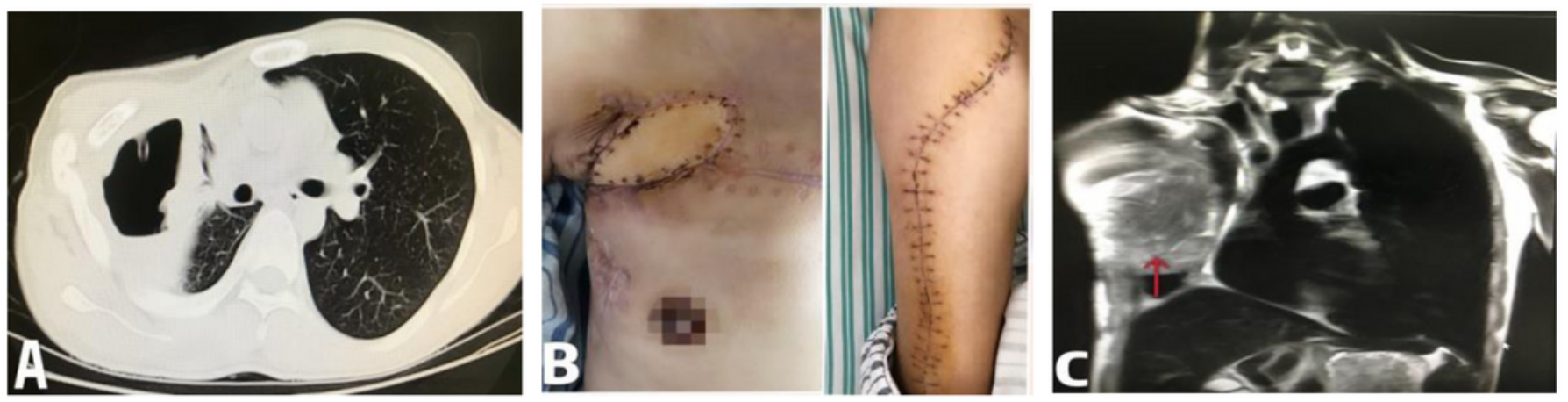
Figure 2

Clinical images of patient-2 A. The chest computed tomography at admission showed right hydropneumothorax with drainage tube placed in the right thoracic cavity. B. The myocutaneous flap was active, and the incision healed well after surgery. $C$. The chest magnetic resonance imaging indicated that the empyema cavity and bronchopleural fistula were successfully blocked (red arrow).

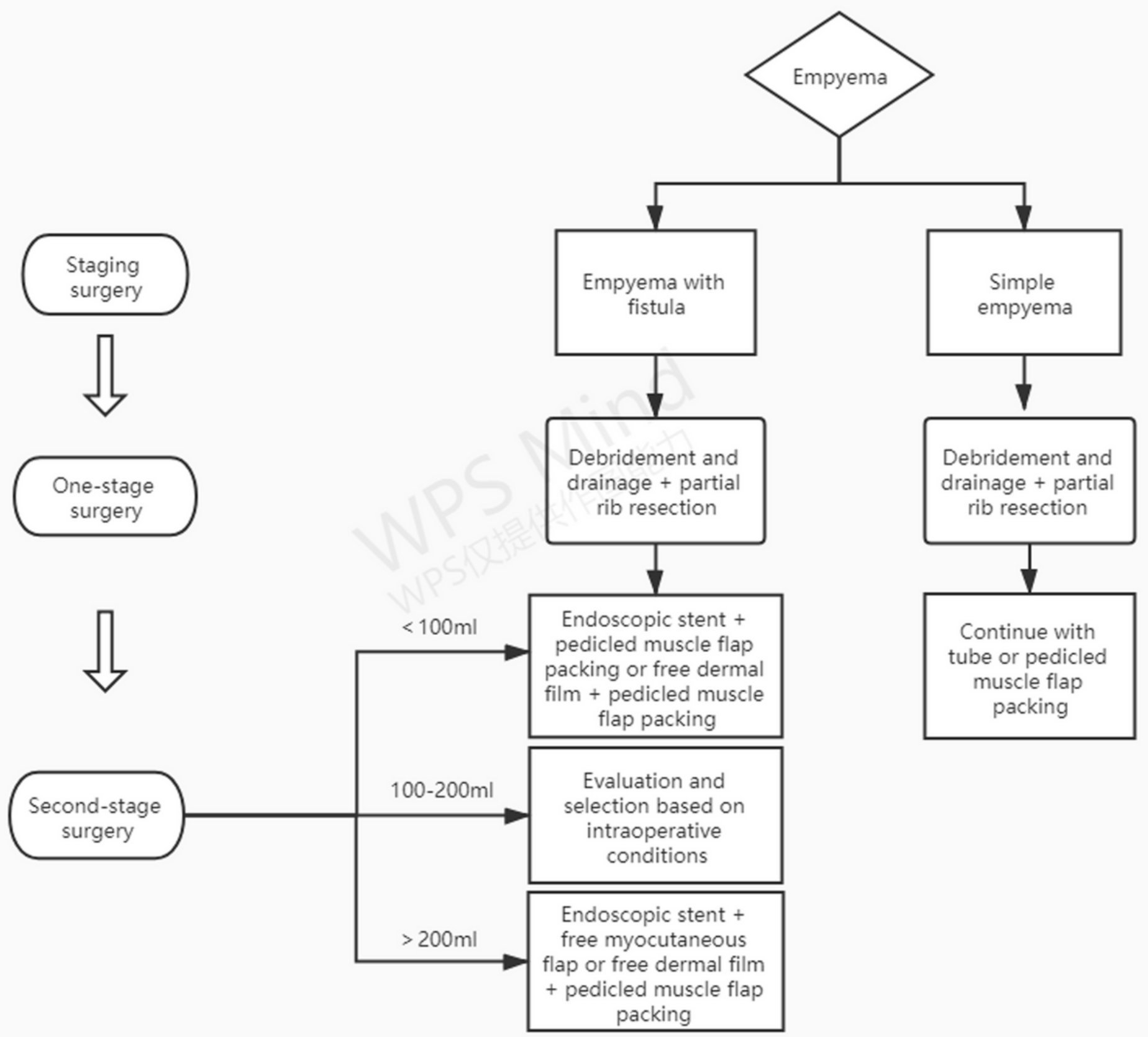

Figure 3

The staging operation flowchart was used to design treatment plans according to the different conditions of the patients. 